\title{
Reliability of Testing of the Electrical Activity of Muscles during Isometric Contractions in Archery
}

\author{
Ihor Zanevskyy ${ }^{1, *}$, Romana Sirenko², Oksana Shukatka², Natalia Bashavets ${ }^{3}$, Ivan Rybchych ${ }^{4}$ \\ ${ }^{1}$ Department of Informatics and Kinesiology, Lviv State University of Physical Culture, Lviv, 79007, Ukraine \\ ${ }^{2}$ Department of Physical Education and Sports, Ivan Franko Lviv National University, Lviv, 79000, Ukraine \\ ${ }^{3}$ Institute of Physical Education, Sports and Rehabilitation, South Ukrainian National Pedagogical University named after KD \\ Ushynsky, Odesa, 65000, Ukraine \\ ${ }^{4}$ Department of Sport Medicine, Human Health, Lviv State University of Physical Culture, Lviv, 79007, Ukraine
}

Received February 5, 2021; Revised April 29, 2021; Accepted May 16, 2021

\section{Cite This Paper in the following Citation Styles}

(a): [1] Ihor Zanevskyy, Romana Sirenko, Oksana Shukatka, Natalia Bashavets, Ivan Rybchych, "Reliability of Testing of the Electrical Activity of Muscles during Isometric Contractions in Archery," International Journal of Human Movement and Sports Sciences, Vol. 9, No. 3, pp. 543 - 553, 2021. DOI: 10.13189/saj.2021.090321.

(b): Ihor Zanevskyy, Romana Sirenko, Oksana Shukatka, Natalia Bashavets, Ivan Rybchych (2021). Reliability of Testing of the Electrical Activity of Muscles during Isometric Contractions in Archery. International Journal of Human Movement and Sports Sciences, 9(3), 543 - 553. DOI: 10.13189/saj.2021.090321.

Copyright $\odot 2021$ by authors, all rights reserved. Authors agree that this article remains permanently open access under the terms of the Creative Commons Attribution License 4.0 International License

\begin{abstract}
BACKGROUND. The archery sport needs a high level of accuracy and coordination between muscle forces. Electromyography is a useful and effective method in studding the electrical activity of muscles in archery. Resulting in a great variation in EMG data repeatability, the results regarding a theory of test reliability should be studied. PURPOSE. The research aims to develop a method of determination concerning reliability of testing of the electrical activity of muscles during isometric contractions in archery. METHODS. The electrical activity of rectus femoral muscle during isometric contractions with the maximal effort in bending of a thigh from $-15^{\circ}$ to $70^{\circ}$ is tested in a sample of twenty skilled male archers of 23-28 years old. ANOVA for repeated measures, ICC and regression models are used. RESULTS. The electrical potential is found to be maximal $(7.15 \mathrm{mV})$, when the joint angle equals $-8.9^{\circ}$ and minimal $(1.44 \mathrm{mV})$, when the joint angle equals $62.8^{\circ}$. A ratio of sum of squares due interaction between subjects' and joint angles' variability to the total sum of squares variability of all measures is accepted as a measure of the error in a hypothetical function of dependence of the electrical activity of muscles on joint angles $(23.3 \%, p<0.001)$. A single measure test after elimination of trends in test-retest results shows a sufficient level of reliability: $\operatorname{ICC}(1,1)=0.745$. Corresponding mean scores reliability of the test with five trials is estimated as good:
\end{abstract}

ICC $(1, k)=0.936,(k=5)$. CONCLUSION. A method of determination concerning reliability of testing of the electrical activity of muscles during isometric contractions specifically to the archery sport is recommended to the practical employment.

Keywords Archery, Electromyography, Reliability, Modeling

\section{Introduction}

The archery sport needs a high level of accuracy and coordination between muscle forces. Specifically, postural stability is required and should be obtained with antagonistic equilibrium of lower extremity and trunk muscles. In this area, Sarro, Viana, and De Barros [1] investigated correlation between postural stability and consistent of arrow hits at the archery target. The main attention regarding scientific research of muscle interaction in archery is given to the upper limbs, specifically to muscles of a forearm. In measuring electrical activity in the forearm muscles (flexor digitorum superficialis, extensor digitorum, deltoid, and trapezius), Tinazci [2] surface electromyography techniques were used. Surface EMG is recognized as a reliable and 
effective method for studying the static contractions in maximal and sub maximal regimes of physical exercises that is typical for the recurve archery [3, p. 87]. From the muscle activity point of view, "the sport of archery requires extreme precision, upper body strength and endurance" [4, p. 539].

Soylu, Ertan, and Korkusuz discovered significant correlation between the repeatability of linear envelopes of surface electromyography of extensor digitorum and flexor digitorum superficialis muscles evaluated as variance ratio and International Federation of Archery (FITA) scores [5, p. 767]. Because of the fact that this correlation was more significant among skilled and beginner archers that non-archers that made possible to use it in assessing archers' sport level, their progress and selection in recruiting to the sort of archery. Using surface electromyography, Suwarganda, Razali, Wilson, and Pharmy studied influence of muscle activity and its variation (bilateral triceps, left deltoid, and right trapezius) on the archery score and a speed of an arrow release. Corresponding individual parameters and linear as well as curvilinear associations were found [6].

Testing of the electrical activity of muscles is a popular method for improving the physical training programs. Values of a thigh angle for the maximum electrical activity of the lower limb muscles were determined regarding the static efforts [7]. A model of measurements of the neuromusculoskeletal movements is based on the two postulates. Muscle constrains are modelled with mechanical forces, which produce force torques relatively axes of joints. Muscle constrains are controlled by neural signals. Buchanan, Lloyd, Manal, and Besier [8, p. 367] described a scheme model of measurements of neuromusculoskeletal movements that contain four consequent phases. During the first phase, a control signal activates a muscle to the aimed level. This activate signal is modelled in binary system of measurements as zero and one. On the second phase, the muscle activation transforms to the muscle contraction that should be measured as mechanical forces. The third phase is dedicated to geometrical measurements and determination of the muscle torques. And on the fourth phase, a second problem of dynamics is being solved, i.e. movements of body segments are determined using muscle moments mechanical properties of the musculoskeletal system. Because of the fact that geometry of the human body and values of relative movements of its segments, the problem is considered in the statement.

The influence of weather conditions on the effectiveness of sport performance play taking into consideration plantar flexor muscular neuromuscular activity was investigated by Girard, Nybo, Mohr, and Racinais [9, p. 154]. A maximal force of the lower segment was measured in cold and hot conditions. Muscle activity was measured as electromyogram test. They showed good validity of the test regarding soleus EMG and maximal muscular forces.

Electrodiagnostics specifically refers to electromyography testing and nerve studies. Electrodiagnostics is not performed in isolation, but is guided by the patient's history and is used as an extension of the clinical examination. The following aspects of electrodiagnostics in the context of back pain were discussed by Sandoval [10, p. 420]: objectives, patient selection, general principles, findings in common pain conditions, sensitivity and specificity, preoperative benefits, and limitations of electrodiagnostics.

Skeletal muscles in activation produced difference constrains. Behm, Whittle, Button, and Power determined differences between skeletal muscles on electrical stimulation regarding maximal static force. The highest constrains showed quadriceps in voluntary torque amplitude. Plantar flexors, elbow flexors, and dorsiflexors activated up to $15 \%$ lower value of force than quadriceps $[11$, p. 236].

It has become quite common to evaluate local muscle activity by means of surface electromyographic signal processing. A large number of studies have been performed yielding signal-based quantitative criteria of fatigue in primarily static but also in dynamic tasks. The non-invasive nature of this approach has been particularly appealing in areas like ergonomics and occupational biomechanics, to name just the most prominent ones. Time domain, frequency domain, time-frequency and time-scale representations, as well as other methods such as fractal analysis and recurrence quantification analysis are described succinctly and are illustrated with their biomechanical applications, research or clinical alike. The future of this methodology is projected by estimating those methods that have the greatest chance to be routinely used as reliable muscle fatigue measures $[12, \mathrm{p}$. 327].

As rule, muscle activity is studied using surface electromyography. Commonly, as a result of measurements maximal and mean values of EMG are taken into consideration [13, p. 102]. So, electromyography is a useful and effective method of studding the electrical activity of muscles in different physical exercises and sports. In the archer's sport, it is commonly used for studding electrical activity of the upper limb muscles' activity and no reports regarding lower limb extremity muscles were noticed in the sport science publications. Therefore, there are no results about reliability of testing corresponding muscles' activity regarding archery.

\section{Objectives}

Because the great variations regarding repeatability of the data are gotten, the results should be studied in the frames of theory of test reliability using intra-class 
correlation model [14]. Therefore, the aim of the research was to develop a method of determination concerning reliability of testing of the electrical activity of muscles during isometric contractions in archery. The purposes of the research were as follow: to determine dependence of the electrical activity of muscle on the joint angle; to evaluate accuracy of the hypothetical function of the electrical activity on the joint angle; to study intraclass correlation test score reliability.

\section{Materials \& Methods}

\subsection{Participants}

A sample of 20 male skilled archers in the age of 23-28 years old was involved into the research. Their body length was $179 \pm 2.6 \mathrm{~cm}(\mathrm{M} \pm \mathrm{SD})$ and body mass $72 \pm 1.9 \mathrm{~kg}$. During the testing, the subjects were in the normal health status. The study complied with the Declaration of Helsinki. Each participant voluntarily provided written informed consent before participating. This study was approved in advance by the ethical committee of Lviv State University of Physical Culture.

\subsection{Procedure}

Electrical activity of rectus femoral muscle during isometric contractions with the maximal effort was tested. Measurements were done using a computer electricneuromyograph M-Test produced by DX Systems [15]. Electrodes $\mathrm{Ag} / \mathrm{AgCL}$ Skintact easitabs RT34 with an adhesive base were used [16]. The electrodes were situated with a free inter-electrode distance. The active electrode was fixed at the innervations zone, and the referential electrode - at the proximal part of a sinew area. The earth electrode was located at the distal part of another extremity. EMG was recorded and analyzed regarding the maximal potential of the muscle electrical activity.

Testing was conducted in the afternoon. The test consisted in bending of thigh in standing position on a special mechanical simulator designed by Korolinska and Sirenko [7]. Subjects were asked to test a preferred extremity on their own decision. The extremity of the subjects used was their dominate one. EMG was recorded at maximum effort in six positions of the thigh situated with joint angles as followed: $-15,0,20,40,50$, and 70 degree. Another testing was done in the average position of the thigh with the angle equal 16.2 degree. Five successive trials with a few minutes break for relax were conducted.

\subsection{Statistical Analysis}

A fifth order algebraic polynome was used as an approximation function of dependence of the electrical activity of the muscle on the joint angle. ANOVA for repeated measures was used to approbate this function. Shapiro - Wilk test was used to evaluate similarity of the electrical muscle potential distribution with the normal distribution.

One Way ANOVA with repeated measures was used to compare variations between subjects and within subjects regarding test scores reliability of the electrical activity in muscles. Corresponding mean score intraclass correlation coefficient (ICC) was calculated using the formula as follows [17]:

$$
\operatorname{ICC}(1, k)=\frac{M S_{B}-M S_{W}}{M S_{B}},
$$

where $k$ is a number of trials, $M S_{B}$ is mean square of scores between persons, and $M S_{W}$ - within persons.

Test reliability of a single trial was calculated using the advanced formula as follows:

$$
\operatorname{ICC}(1,1)=\frac{M S_{B}-M S_{W}}{M S_{B}+(k-1) M S_{W}} .
$$

A coefficient of linear regression as a model of trends in repeated measures (test-retest) was calculated using a formula as follows [18]:

$$
b=6 \frac{\frac{2}{k+1} \sum_{j=1}^{k} j \bar{x}_{j}-\sum_{j=1}^{k} \bar{x}_{j}}{k(k-1)} .
$$

where $j=1,2, \ldots k$ are numbers of trials, $\bar{x}_{j}$ are mean scores of a group in the test trials. The significance of the regression coefficient was estimated using t-test with $(k-$ 2) degrees of freedom:

$$
t=b \sqrt{\frac{k\left(k^{2}-1\right)(k-2)}{12 \sum_{j=1}^{k}\left(y_{j}-\bar{x}_{j}\right)^{2}}},
$$

where $y_{j}=a+b j$ is the approximate mean score in the $j^{\text {th }}$ trial, $a=\bar{x}_{T}-b \frac{k+1}{2}$ is a free member in the equation of regression, $\bar{x}_{T}$ is a total mean score.

The accuracy of approximation was determined using the coefficient of determination:

$$
R^{2}=\frac{\sum_{j=1}^{k}\left(y_{j}-\bar{x}_{T}\right)^{2}}{\sum_{j=1}^{k}\left(\bar{x}_{j}-\bar{x}_{T}\right)^{2}} .
$$

Calculations were done using Statistica 
(www.statsoft.com), Mathematica (www.wolfram.com), fifth power $(s=6)$ : and MathCAD (www.mathcad.com) computer programs.

\section{Results}

\subsection{Dependence of the Electrical Activity of Muscle on the Joint Angle}

In average, the electrical activity of rectus femoral muscle was found from 6.59 to $6.69 \mathrm{mV}$ as the joint angle increases from $-15^{\circ}$ to $0^{\circ}$, and then the electrical activity decreases approximately twice and a half (to $2.69 \mathrm{mV}$ ) when the joint angle increases up to $70^{\circ}$ (Table 1). Relative dispersion of the sample values is approximately constant in the whole interval of joint angle testing: the ratio of mean value and standard deviation equals $4.0 \pm 0.1$ (Figure 1). There is a strong correlation between these statistics $(r=0.996, p<0.001)$.

Due to the fact that the electrical activity of the muscle was tested for six values of the joint angle, the approximation function was estimated as a polynome of a

$$
u(\vartheta)=\sum_{J=0}^{s-1} a_{J} \vartheta^{J}
$$

where $u(\vartheta)$ is the maximal electrical potential, $\vartheta$ is a thigh joint angle, $a_{J}$ are coefficients of the polynome, $J=0,1,2 \ldots(s-1)$.

Coefficients of the polynome were determined as a solution to a system of linear algebraic equations:

$$
\sum_{J=0}^{s-1} a_{J} \vartheta_{j}^{J}-u\left(\vartheta_{j}\right)=0,
$$

where $j=1,2 \ldots s$ are numbers of joint angles, $\vartheta_{j}=-15$, $0,20,40,50,70^{\circ}$ are joint angles.

The system (7) was solved according to Cramer's Rule using MathCAD, and the coefficients were calculated as follows: $a_{0}=6.6900, \quad a_{1}=-8.1084 \mathrm{E}-2, \quad a_{2}=$ $-6.0739 \mathrm{E}-4, \quad a_{3}=2.2837 \mathrm{E}-4, \quad a_{4}=-7.1926 \mathrm{E}-6, a_{5}=$ $5.8914 \mathrm{E}-8$.

\begin{tabular}{|c|c|c|c|c|c|c|}
\hline \multirow{2}{*}{ Statistics* } & \multicolumn{6}{|c|}{ Joint angle (degree) } \\
\hline & -15 & 0 & 20 & 40 & 50 & 70 \\
\hline $\operatorname{Max}$ & 10.13 & 10.70 & 7.05 & 7.23 & 2.47 & 3.62 \\
\hline Min & 3.77 & 2.80 & 2.36 & 3.10 & 1.89 & 2.71 \\
\hline$M$ & 6.59 & 6.69 & 5.69 & 4.71 & 3.12 & 2.69 \\
\hline$S D$ & 1.65 & 1.70 & 1.35 & 1.19 & 0.83 & 0.68 \\
\hline$S W-W$ & 0.974 & 0.957 & 0.954 & 0.977 & 0.956 & 0.952 \\
\hline$p$ & 0.835 & 0.496 & 0.427 & 0.893 & 0.473 & 0.403 \\
\hline
\end{tabular}

Table 1. Electrical activity of the muscle $(\mathrm{mV})$ on joint angle $(n=20)$

*Descriptions of symbols are in Appendix section

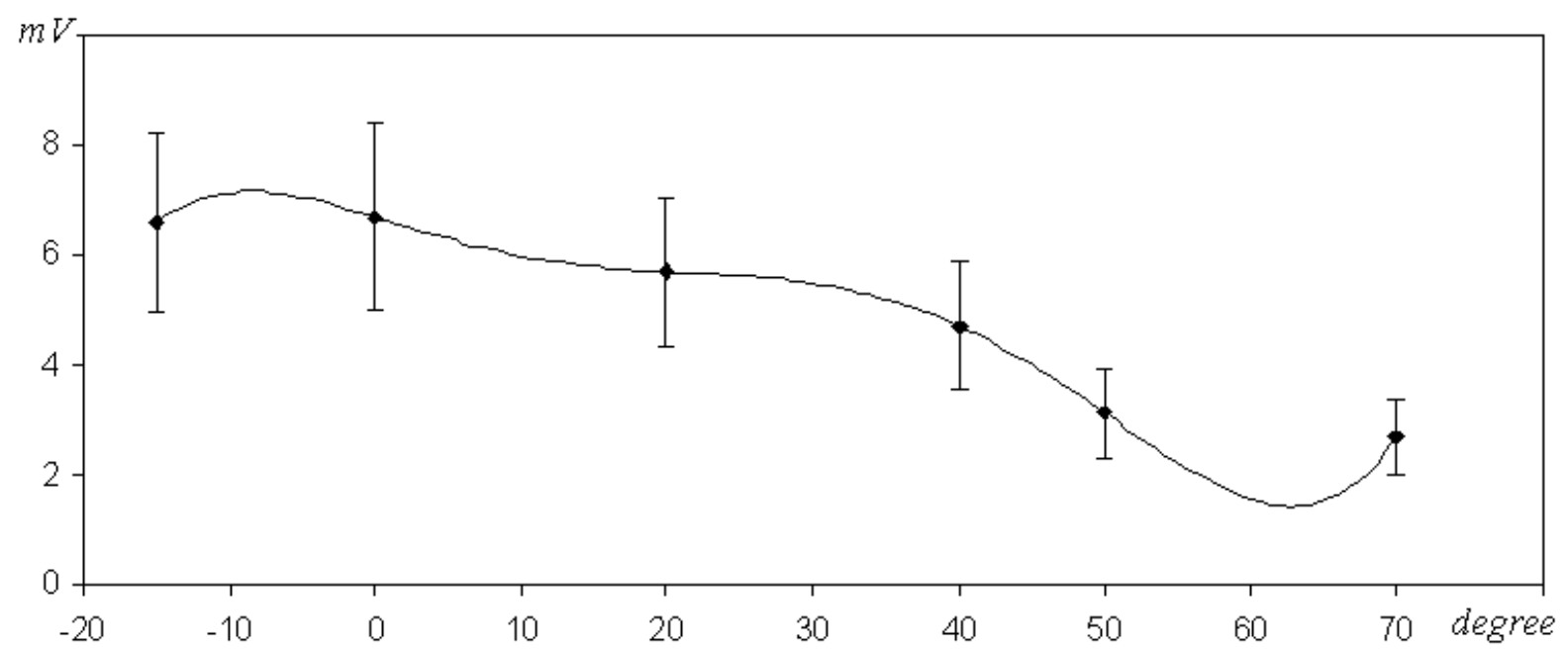

Figure 1. Average potential of the electrical activity of the muscle $(M \pm S D)$ as a function of the joint angle $(n=20)$ 
In general, maximal or minimal values of the function (6) can be situated at the boundaries of the diapason $\left(\vartheta_{1}=\right.$ $-15^{\circ}$ and $\left.\vartheta_{6}=70^{\circ}\right)$ and at the extreme points. The function has extreme values, when its first derivation

$$
\sum_{J=0}^{s-1} a_{J} \vartheta_{j}^{J}-u\left(\vartheta_{j}\right)=0
$$

equals zero. If the second derivation

$$
\frac{\partial^{2} u(\vartheta)}{\partial \vartheta^{2}}=\sum_{J=2}^{s-1} J(J-1) a_{J} \vartheta^{J-2},
$$

is negative, the function is maximum, and if - positive, the function is minimum.

The first derivation (8) equals zero when $\vartheta=-8.9^{\circ}$ and $62.8^{\circ}$. Because when $\vartheta=-8.9^{\circ}$, the second derivation (9) is negative $\left(-20.8 \mu \mathrm{V} /{ }^{\circ} /{ }^{\circ}\right)$, the function (6) is maximum (7.15 mV). When $\vartheta=62.8^{\circ}$, the second derivation (9) is positive $\left(22.7 \mu \mathrm{V} /{ }^{\circ} /{ }^{\circ}\right)$, therefore, the function is minimum $(1.44 \mathrm{mV})$. On the boundaries $\left(\vartheta_{\min }=-15^{\circ}\right.$ and $\vartheta_{\max }=$ $\left.70^{\circ}\right)$, the function values $(6.59$ and $2.69 \mathrm{mV})$ are not maximum or minimum.

An average potential of the electrical activity was determined as a value of a function with argument $\left(\vartheta_{a v}\right)$ calculated as a solution of the equation bellow:

$$
I\left(\vartheta_{a v}\right)=1 / 2 I\left(\vartheta_{\max }\right)
$$

where $I(\vartheta)=\int_{\vartheta_{\min }}^{\vartheta} u(\varsigma) d \varsigma$.

After replacing integrals with sums: $I(\vartheta)=\sum_{J=0}^{s-1} \frac{a_{J}}{J+1}\left(\vartheta^{J+1}-\vartheta_{\min }^{J+1}\right)$, algebraic equation of $s^{\text {th }}$ power was derived: $\sum_{J=0}^{s-1} \frac{a_{J}}{J+1}\left(\vartheta_{\max }^{J+1}+\vartheta_{\min }^{J+1}-2 \vartheta_{a v}^{J+1}\right)=0$. Given, no solution in radicals of this algebraic equation of a sixth power, numerical method of solving was used. The solution was gotten with Solve program of Mathematica package, as follows: $\vartheta_{a v}=16.2^{\circ}, u\left(\vartheta_{a v}\right)=5.76 \mathrm{mV}$.

\subsection{Accuracy of the Hypothetical Function of the Electrical Activity on the Joint Angle}

Including the fact that statistical hypothesis regarding normal distribution in samples results of measurements was not rejected $(S W-W=0.945-0.977, p=$ 0.302-0.893), parameter statistics were used (see Table $1)$.

Mean values in the group of subjects are the best estimation of the electrical activity of the muscle as a function of joint angles. The error of this function was estimated as a part of sum of squares interaction between subjects and between joint angles in a total sum of squares between all the results of measurements:

$$
Q_{E}=\frac{S S_{E}}{S S_{T}},
$$

where $S S_{E}$ is a sum of squares interaction between subjects and joint angles, $S S_{T}$ is a total sum of squares between all the results of measurements.

A family of linearly correlated functions $\left(x_{i}\right)$ was studied:

$$
x_{i}=f(\vartheta)+C_{i},
$$

where $f(\vartheta)$ is the hypothetical function of the electrical activity of the muscle defined between boundary values of joint angles $\theta_{\min }, \theta_{\max }, C_{i}$ are constants. If $\bar{C}=0, f(\vartheta)=u(\vartheta)$ like the function (6).

A sum of squares due to subjects and joint angles interacted variation is defined with the equation as follows:

$$
S S_{E}=S S_{T}-S S_{C}-S S_{R},
$$

where $S S_{C}$ is a sum of squares variation between joint angles (columns), $S S_{R}$ is a sum of squares variation between subjects (rows).

A sum of squares variation between joint angles was calculated with the equation as follows:

$$
S S_{C}=\sum_{i=1}^{n} \int_{\vartheta \min }^{\vartheta \max }\left[\bar{x}(\vartheta)-\bar{x}_{T}\right]^{2} d \vartheta=n \int_{\vartheta \min }^{\vartheta_{\max }}[f(\vartheta)-\bar{f}]^{2} d \vartheta,
$$

where $\bar{x}(\vartheta)=\frac{1}{n} \sum_{i=1}^{n}\left[f(\vartheta)+C_{i}\right]=f(\vartheta)+\bar{C}, \bar{C}=\frac{1}{n} \sum_{i=1}^{n} C_{i}$ are sample means for the joint angle and constants,

$$
\begin{gathered}
\bar{x}_{T}=\frac{\sum_{i=1}^{n} \int_{\vartheta \text { min }}^{\vartheta_{\max }\left[f(\vartheta)+C_{i}\right] d \vartheta}}{n\left(\vartheta_{\max }-\vartheta_{\min }\right)}=\bar{f}+\bar{C} \text { is a total mean, } \\
\vartheta_{\max } f(\vartheta) d \vartheta \\
\bar{f}=\frac{\int_{\text {min }} f\left(\vartheta_{\text {min }}\right.}{\vartheta_{\text {max }}-\vartheta_{\text {min }}} \text { is a mean of the hypothetical function. }
\end{gathered}
$$

A sum of squares between subjects was calculated with the equation as follows:

$$
\begin{aligned}
& S S_{R}=\sum_{i=1}^{n} \int_{\vartheta \min }^{\vartheta_{\max }}\left(\bar{x}_{i}-\bar{x}_{T}\right)^{2} d \vartheta= \\
& \sum_{i=1}^{n} \int_{\vartheta_{\min }}^{\vartheta_{\max }}\left(C_{i}-\bar{C}\right)^{2} d \vartheta=\left(\vartheta_{\max }-\vartheta_{\min }\right) \sum_{i=1}^{n}\left(C_{i}-\bar{C}\right)^{2}
\end{aligned}
$$

where $\bar{x}_{i}=\frac{\int_{\vartheta_{\min }}^{\vartheta_{\max }}\left[f(\vartheta)+C_{i}\right] d \vartheta}{\vartheta_{\max }-\vartheta_{\min }}=\bar{f}+C_{i}$ is a sample mean for the $i^{\text {th }}$ subject.

A total sum of squares variation was calculated with the equation as follows: 


$$
\begin{aligned}
& S S_{T}=\sum_{i=1}^{n} \int_{\vartheta \min }^{\vartheta \max }\left[x(\vartheta)-\bar{x}_{T}\right]^{2} d \vartheta= \\
& \sum_{i=1}^{n} \int_{\vartheta \min }^{\vartheta \max }\left[f(\vartheta)-\bar{f}+C_{i}-\bar{C}\right]^{2} d \vartheta= \\
& n \int_{\vartheta \max }^{\max _{\min }[f(\vartheta)-\bar{f}]^{2} d \vartheta+\left(\vartheta_{\max }-\vartheta_{\min }\right) \sum_{i=1}^{n}\left(C_{i}-\bar{C}\right)^{2}}, \\
& +2 \sum_{i=1}^{n}\left(C_{i}-\bar{C}\right) \int_{\vartheta \min }^{\int_{\max }}[f(\vartheta)-\bar{f}] d \vartheta
\end{aligned}
$$

Taking into account that $\sum_{i=1}^{n}\left(C_{i}-\bar{C}\right) \equiv 0$ or that $\int_{\vartheta \min }^{\vartheta \operatorname{mqx}}[f(\vartheta)-\bar{f}] d \vartheta \equiv 0$, and comparing $(14,15)$ with $(16)$, one can notice that $S S_{T}=S S_{C}+S S_{R}$, i.e. $S S_{E}=0$. Therefore, one can conclude that for the linear correlated functions (12) a sum of squares interaction between subjects and joint angles equals zero. If some correlation between rows and columns occurs, corresponding sum of squares differs of zero. The value of this sum of squares relatively to the total sum of squares variation was used as a measure of the error of a mean value estimation of electrical activity in the function of the joint angle.

Because the function of electrical activity as a result of measurements is interrupted and is defined in six points, the integrals in (14-16) were substituted with sums:

$$
\begin{gathered}
S S_{C}=n \sum_{j=1}^{S}\left(\bar{x}_{j}-\bar{x}_{T}\right)^{2}, S S_{R}=s \sum_{i=1}^{n}\left(\bar{x}_{i}-\bar{x}_{T}\right)^{2}, \\
S S_{T}=\sum_{i=1}^{n} \sum_{j=1}^{S}\left(x_{i j}-\bar{x}_{T}\right)^{2},
\end{gathered}
$$

where $\bar{x}_{i}=\frac{1}{s} \sum_{j=1}^{s} x_{i, j}, \quad \bar{x}_{j}=\frac{1}{n} \sum_{i=1}^{n} x_{i, j}, \quad \bar{x}_{T}=\frac{1}{n} \sum_{i=1}^{n} \sum_{j=1}^{s} x_{i, j}$.
Corresponding results of ANOVA on the electrical activity in different join angle are collected in Table 2.

The main income of variation to the total sum of squares is done thanks to the variation caused by changing of the joint angle $\left(Q_{\mathrm{C}}=60.9 \%\right)$. The results show non-significant interaction between variation in persons and joint angles. Corresponding income of variation in the total sum of squares is smaller than a quarter $\left(Q_{\mathrm{E}}=\right.$ $23.3 \%$ ). This conclusion was supported with a ratio of the person's variance $\left(F_{\mathrm{R}}=3.40\right)$ and the angle's variance $\left(F_{\mathrm{C}}\right.$ $=49.63)$ to the error's variance, which were considered on the strong significant level $(p<0.001)$. One can conclude a high level of similarity between the characters of individual functions of dependence of the electrical activity in the muscle on the joint angle. Therefore, the average values (1) in a sample could be used as a sufficient estimation of the electrical activity in the muscle.

\subsection{Intraclass Correlation Test Score Reliability}

Reliability of a test with a single trial (relatively to the real scores of the first trial) was assumed equal to the mean score reliability estimated the virtual data with reduction of the mean score reliability from $k$ trials to one trial. A total trend in scores between trials was eliminated under the corresponding condition as follows:

$$
\bar{X}_{T}=\bar{x}_{i, 1}(\mathrm{mV}) \text {, }
$$

A formula of the scores transformation was derived as followed:

$$
X_{i, j}=x_{i, j}-b(j-1)+c
$$

where $\bar{X}_{T}$ is a total mean of the transformed scores, $\bar{x}_{i, 1}$ is a real mean score of a sample in the first trial.

where $x_{i, j}$ are individual scores of repeated trials, $X_{i, j}$ are individual transformed scores, $c$ is a constant regarding (18), the regression coefficient $b$ was calculated using (3).

Table 2. ANOVA results on the electrical activity of the muscle in different join angle

\begin{tabular}{|c|c|c|c|c|c|c|c|}
\hline Source of variation & $S S$ & $d f$ & $M S$ & $F$ & $p$ & $F_{0,05}$ & $Q \%$ \\
\hline Persons (Rows) & 76.8 & 19 & 4.04 & 3.40 & $<0.001$ & 1.70 & 15.8 \\
\hline Angles (Columns) & 295.3 & 5 & 59.07 & 49.63 & $<0.001$ & 2.31 & 60.9 \\
\hline Errors (R×C) & 113.1 & 95 & 1.19 & - & - & - & 23.3 \\
\hline Total & 485.3 & 119 & & - & - & - & 100.0 \\
\hline
\end{tabular}


The members of (19) were summarized in rows and columns: $\sum_{i=1}^{n} \sum_{j=1}^{k} X_{i, j}=\sum_{i=1}^{n} \sum_{j=1}^{k} x_{i, j}-\frac{b}{2}(k-1) k n+c k n$, and then divided with a total number of scores $(k n)$ :

Mean scores of a group in test trials after the transformation (19) were derived from equation $\sum_{j=1}^{k} X_{i, j}=\sum_{j=1}^{k} x_{i, j}-\frac{b}{2}(k-1) k+c k$ as follows:

Statistics of the repeated measures (five times testretest) of the electrical potential for the average thigh angle were collected in Table 3. Parameters of linear regression and of the transformation equation were calculated using formulas (8) and (21): $b=-0.3004, a=$ 6.337, $R^{2}=88.4 \%$ (Figure 2). A constant in the transformation formula (19) was calculated as $c=-0.1718$ using (21), and t-test with $(k-2)$ degrees of freedom showed rather strong significance level for the slope coefficient $(t=4.78, p=0.017)$. Elimination of the trends causes reduction of trial-to-trail variation:

$$
\bar{X}_{T}=\bar{x}_{T}-\frac{b}{2}(k-1)+c .
$$

Taking into account (18), a constant in the transformation formula (19) was determined from (20) as follows:

$$
\begin{gathered}
c=\bar{x}_{i, 1}+\frac{b}{2}(k-1)-\bar{x}_{T} . \\
\bar{X}_{j}=\bar{x}_{j}-b \frac{k-1}{2}+c . \\
n \sum_{j=1}^{k}\left[\left(\bar{x}_{j}-\bar{x}_{T}\right)^{2}-\left(\bar{X}_{j}-\bar{X}_{T}\right)^{2}\right]=n b^{2} \frac{k}{12}\left(k^{2}-1\right)=18.0(\mathrm{mV})^{2}
\end{gathered}
$$

This is an absolute measure of variation caused by the learning effect. The difference of sum of squares should be evaluated relatively to a total sum of squares variation. The relative measure of the trends' effect component was calculated as a difference of a part of sum of squares within subjects' variation: $Q_{x W}-Q_{X W}=25.1-21.2=3.9 \quad(\%)$. activity in the muscle.

Because all the five samples showed distribution similar to normal $(S W-W=0.945-0.975, p=0.302-0.848)$, one way ANOVA was used in estimation of test score reliability (Table 4).

Mean scores reliability of the test with five trials was calculated with real scores $\left(x_{i, y}\right)$ using formula (1). Rather good reliability was estimated with the value of this intra class correlation coefficient: $\operatorname{ICC}(1, k)=0.921$. Corresponding mean scores reliability of the test after eliminating trends of mean scores in trial after trial was calculated using transformed scores $\left(X_{i, y}\right)$ and the same formula (1). Some better reliability was estimated with this hypothetical value of the correlation coefficient: $\operatorname{ICC}(1, k)=0.936$. Reliability of a single trial test was calculated using transformed scores and the formula (2): $\operatorname{ICC}(1, k)=0.745$. This value of the correlation coefficient made it possible to estimate test reliability on the sufficient level. It is interesting, that the same coefficient calculated with a real data showed questionable reliability of the test: $\operatorname{ICC}(1, k)=0.698$.

Table 3. Test scores (x), transformed data (X)

\begin{tabular}{|c|c|c|c|c|c|}
\hline \multirow{2}{*}{$\begin{array}{c}\text { Statistics } \\
(n=20)\end{array}$} & \multicolumn{5}{|c|}{ Trials $(j)$} \\
\cline { 2 - 6 } & 1 & 2 & 3 & 4 & $k=5$ \\
\hline $\bar{x}_{j}$ & 5.87 & 5.80 & 5.62 & 5.25 & 4.64 \\
\hline$x_{j}$ (max) & 8.35 & 9.67 & 8.97 & 8.61 & 8.60 \\
\hline$x_{j}$ (min) & 2.12 & 3.36 & 2.20 & 1.62 & 0.38 \\
\hline $\bar{X}_{j}$ & 5.69 & 5.93 & 6.05 & 5.98 & 5.67 \\
\hline$X_{j}(\max )$ & 8.18 & 9.80 & 9.40 & 9.34 & 9.63 \\
\hline$X_{j}(\min )$ & 1.95 & 3.49 & 2.62 & 2.35 & 1.41 \\
\hline$S D$ & 1.52 & 1.70 & 1.77 & 1.93 & 2.51 \\
\hline$S W-W$ & 0.945 & 0.959 & 0.969 & 0.975 & 0.952 \\
\hline$p$ & 0.302 & 0.524 & 0.727 & 0.848 & 0.396 \\
\hline
\end{tabular}

\section{Discussion}

Regarding the purpose to develop a method of determination of reliability for testing the electrical activity of muscles during isometric contractions, the research results showed effectiveness of the intraclass correlation test data reliability model that confirms well-known results regarding, for example, the reliability of quadriceps surface electromyography measurements [19, p. 1085]. It was demonstrated, that the original methods used for this purpose should be modified with preliminary elimination of trends in the test - retest measures (see "Intraclass correlation test data reliability" sub-chapter). Corresponding effect of using this method gave more accurate results for evaluation of the test reliability from the questionable level to the good one, i.e. the mark of the test was increased in two levels while a common gradation of test reliability in the human physical activity science and medicine was determined as follows: unacceptable, questionable, acceptable, moderate, good, excellent $[20$, p. 862]. There is a strong correlation $(r=$ $0.996, p<0.001)$ between arithmetic mean and standard deviation values of the electrical potential in rectus femoral muscle at the whole range of thigh joint angle values (from $-15^{\circ}$ to $70^{\circ}$ ) while the ratio of these statistics is approximately constant $(4.0 \pm 0.1)$. The electrical potential was found to be maximal $(7.15 \mathrm{mV})$ when the joint angle equals $-8.9^{\circ}$ and minimal $(1.44 \mathrm{mV})$ when the joint angle equals $62.8^{\circ}$. A ratio of sum of squares due 
interaction between subjects' and joint angles' variability to the total sum of squares variability of all measures was accepted as a measure of the error of a hypothetical function of dependence of the electrical activity of muscles on joint angles $(23.3 \%, p<0.001)$.

Hypothetical elimination of trends in repeated measures of the electrical potential for rectus femoral muscle leaded to a correct conclusion regarding the reliability of the test. A single measure test showed the sufficient level of reliability: $\operatorname{ICC}(1,1)_{X}=0.745$, i.e. significantly better than the acceptable level derived from the real data: $\operatorname{ICC}(1,1)_{x}$ $=0.698$. Corresponding mean data reliability of the test with five trials was estimated as good: $\operatorname{ICC}(1, k)_{x}=0.921$ and $\operatorname{ICC}(1, k)_{X}=0.936$ [21].

Similar results on test reliability regarding electromyography indices of fatigue of the back muscle capacity test were evaluated using interrupted static contractions in the frames of the test-retest reliability techniques. An intra-class correlation coefficient was calculated based on the results of (44 males and 29 females (20-55 years old) ranged between 0.75 and 0.83 [22]. These results are rather similar to the results obtained in our research (see subchapter 4.3. Intraclass correlation test score reliability). Postural stability in the sport of archery influences on aiming and arrow release. There is a significant correlation between amplitudes of body sways and archery scores in the skilled recurve archers [23, p. 49]. Postural stability depends on stable muscle activation and coordination, and maximum sway speed. Postural sway and elite archery score performance are in significant relationship $(\mathrm{p}<0.001)$. Furthermore, there are clear relationships between electrical activity of muscles of the upper and lower extremities [24, p.1778]. It was showed that upper and lower limb muscle activation are bidirectionally and ipsilaterally coupled. Sixteen muscles of the upper and lower limbs on fours at each of the extremities during maximal muscle efforts and lower limb muscles were as followed: vastusmedialis, medial hamstrings, tibialis anterior, and soleus. These relationships are an important problem of studding interaction of a bow and an archer body stability interaction. It is interesting to study coactivations in muscles of the lower extremities regarding archer's body stability [25].

Effects of self-paced incline treadmill walking on lower limb muscles activation level were discovered by $\mathrm{Lu}$ and Al-Amri [26]: the muscle activations of biceps femoris and soleus were significantly $(p<0.001)$ increased and decreased during uphill walking and downhill walking, respectively. Whilst soleus activity level was significantly reduced during uphill compared to level and downhill walking. Downhill walking significantly increased muscle activation level of tibialis posterior while it was significantly decreased during uphill walking. These muscles should be a subject of studding their electrical activity during aiming and arrow launch phases.

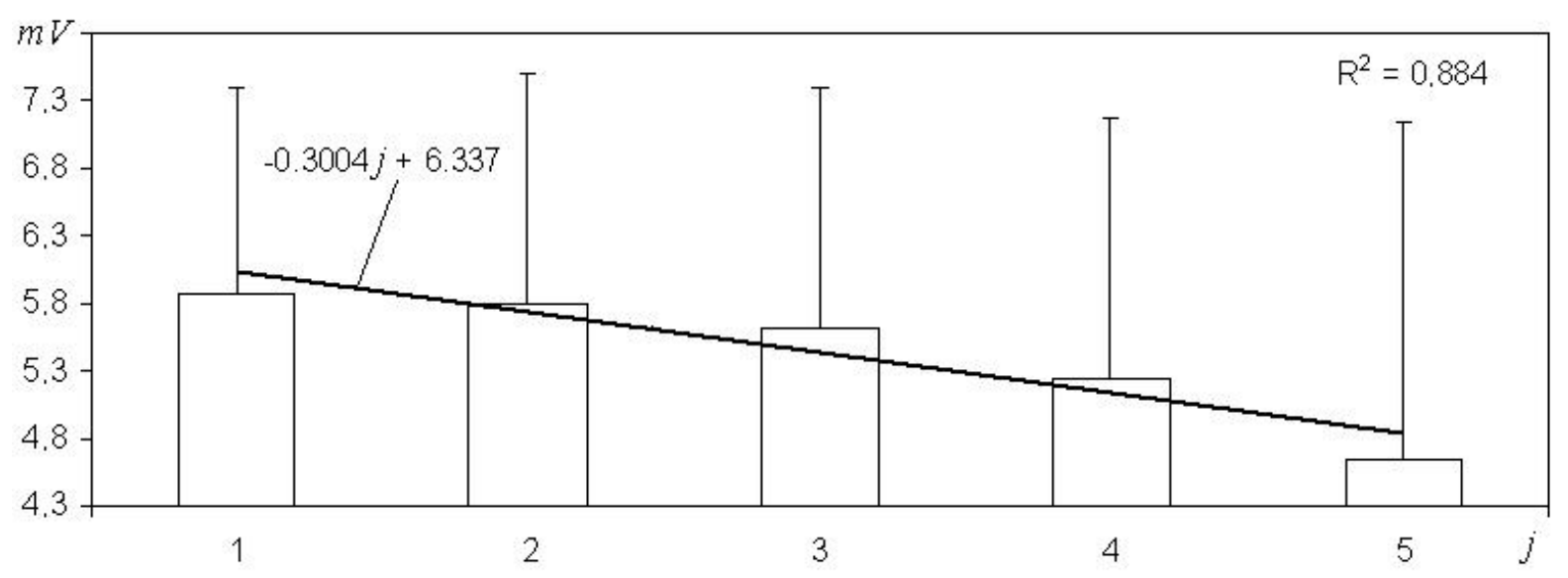

Figure 2. Electrical potential of the muscle $(M+S D)$ during five repeated measures and linear approximation of trends

Table 4. ANOVA results of test scores (upper) and transformed scores (lower)

\begin{tabular}{|c|c|c|c|c|c|c|}
\hline Variation & $S S$ & $d f$ & $M S$ & $F$ & $p \%$ \\
\hline Between (B) & 276.1 & 19 & 14.53 & $\begin{array}{l}12.58^{*} \\
15.64^{*}\end{array}$ & $<0.001$ \\
\hline Within (W) & 92.4 & 80 & 1.15 & - & - \\
Total (T) & 74.4 & 99 & 3.72 & & 21.9 \\
\hline
\end{tabular}


The main attention in the electromyographic study of muscle activity of the archer's body was focused on upper extremities, especially on the bow hand, forearm, and arm. Ertan [27, p. 357] measured muscle activation of the flexor digitorum superficialis and extensor digitorum during two-second period: one second before and one second after the clicker snap. Ertan, Soylu, and Korkusuz [28, p. 222] defined the electromyographic data of the bow forearm as a valid and useful method for evaluation of archers' shooting techniques and a whole sport qualification. They recorded electrical activity of flexor digitorum superficialis and extensor digitorum muscles and compared this data with shooting techniques data. The same investigations are reasonable in future regarding lower limb muscle.

There are some restrictions for application of the method of hypothetical elimination of trends in repeated measures of the electrical potential. A linear function of the hypothetical model of trends was assumed and showed relevant results in the analysis of the test reliability of electrical activity of rectus femoral muscle with the maximal effort in bending of a thigh. Regarding another muscle and/or another exercise the linear function could not be relevant. In this case, nonlinear approximation could be useful. One of the universal functions is polynome of the power equal two and higher, and for the trends caused with learning or getting tired, modelled in the first estimation should be polynome of the second power, i.e. quadratic parabola [29].

Another restriction is caused by specific composition of the sample involved in the testing. The research was dedicated to the sport of archery and high skilled archers were tested there, but the method developed and evaluated in the research is not only specific for the population of high skilled archers but also for the shooting sports exactly. It is significant, as a matter of fact, to any other population regarding studies in different fields of physical activity: education, sports, rehabilitation and so on.

\section{Conclusions}

A method of determination of reliability for testing the electrical activity of muscles during isometric contractions specifically to the archery sport has been developed in this research. A method of hypothetical elimination of trends in repeated measures of the electrical potential in rectus femoral muscle made possible to get a correct conclusion regarding reliability of the test. The methodological approach prepared in this article for studying the electrical activity of muscles could be proposed for application of the method for determination of sport test reliability in other fields of Sports Sciences, for example in determination of the test reliability of physical activity questionnaire [30].

Due to the fact that the sport of archery muscles of the upper extremity is involved in execution of the sport exercise, reliability of testing of these muscles should be studied. Future research on this theme would be important, taking into account the absence of correlation between electrical activity of muscles in upper and lower extremities [31]. The research was based on the results of testing the male archers. It should be noted that archery sport is popular among female archers as well as among male archers; corresponding research will be interesting regarding the female samples.

A common algorithm of the practical usage of the method is as follows:

- testing of the electrical activity of the studied muscle and determination of maximal values of the potential, for example EMG recorded at maximum effort in several positions of the body parts situated in the full range of corresponding joint angles,

- ANOVA of measured data (see Table 2) and accepting the situation of corresponding body parts regarding the electrical potential,

- $\quad$ test - retest measures of the electrical activity (three or more times),

- determination of the function of trends appeared during the test - retest trials (linear or another function),

- elimination of trends in the results of the test - retest measurements (19),

- ANOVA of transformed data and comparison of variations between subjects and within subjects regarding test data reliability of the electrical activity in muscles (see Table 4),

- calculation of the intraclass correlation coefficient of reliability $(1,2)$ and evaluation of testing reliability with a single and multiple trials.

\section{Acknowledgements}

The authors are very grateful to archers for their fruitful participation in the research.

\section{Conflict of Interest}

The authors declare no conflict of interest regarding this paper. 


\section{Appendix}

\section{Nomenclature}

\begin{tabular}{|c|c|}
\hline Symbol & Description \\
\hline$C$ & constant \\
\hline$F$ & Fisher - Snedecor parameter \\
\hline$I$ & integral of the electrical potential on the joint angle \\
\hline ICC & intraclass correlation coefficient \\
\hline$J$ & powers of polynome members \\
\hline$M$ & mean value \\
\hline$M S$ & variance \\
\hline$S D$ & standard deviation \\
\hline$R^{2}$ & coefficient of determination \\
\hline$Q$ & part of sum of squares variation \\
\hline$S S$ & sum of squares variation \\
\hline$S W-W$ & Shapiro - Wilk parameter \\
\hline$X$ & transformed data of the test results \\
\hline$a$ & free member of the linear regression equation \\
\hline$a_{J}$ & coefficients of a polynome \\
\hline$b$ & coefficient of linear regression \\
\hline$c$ & constant \\
\hline$d f$ & degree of freedom \\
\hline$f$ & $\begin{array}{l}\text { hypothetical function of the electrical activity of } \\
\text { muscles }\end{array}$ \\
\hline$i$ & numbers of subjects \\
\hline$j$ & numbers of joint angles positions / numbers of trials \\
\hline$k$ & common number of trials \\
\hline$n$ & common number of subjects \\
\hline$p$ & significance \\
\hline$r$ & Pearson interclass correlation coefficient \\
\hline$s$ & $\begin{array}{l}\text { polynome's power and common number of studied } \\
\text { joint angles }\end{array}$ \\
\hline$t$ & Student parameter \\
\hline$u$ & electrical potential \\
\hline$x$ & test results \\
\hline$y$ & linear approximation \\
\hline$\vartheta$ & thigh joint angle \\
\hline
\end{tabular}

\section{REFERENCES}

[1] Sarro K. J., Viana T. C., De Barros R. M. L., "Relationship between bow stability and postural control in recurve archery", European Journal of Sport Science. Published online: 04 May 2020. DOI: 10.1080/17461391.2020.17544 71 .

[2] Tinazci C., "Shooting dynamics in archery: A multidimensional analysis from drawing to releasing in male archers", Procedia Engineering, vol. 13, pp. 290-296, 2011. DOI: $10.1016 /$ j.proeng.2011.05.087.

[3] Lariviere C., Gagnon D., Gravel D., Arsenault A. B., "Toward the development of predictive equations of back muscle capacity based on frequency and temporal-domain electromyographic indices computed from intermittent static contractions". Spine Journal, vol. 9, no. 1, pp. 87-95, 2009. DOI: 10.1016/j.spinee.2007.09.012.

[4] Spratford W., Campbell R., "Postural stability, clicker reaction time and bow draw force predict performance in elite recurve archery". European Journal of Sport Science, vol. 17, no. 5, pp. 539-545, 2017. DOI: 10.1080/17461391.2017.1285963.

[5] Soylu A. R., Ertan H., Korkusuz F., "Archery performance level and repeatability of event-related EMG". Human Movement Science, vol. 25, no. 6, pp. 767-774, 2007. DOI: 10.1016/j.humov.2006.05.002.

[6] Suwarganda E., Razali R., Wilson B., Pharmy A., "Influence of muscle activity on shooting performance in archery: Preliminary findings", 30th Annual Conference of Biomechanics in Sports, Melbourne. pp. 319-322, 2012. DOI: 10.13140/RG.2.1.3843.2160.

[7] Korolinska S.V., Sirenko P.O., "Features interference electromyogram of $\mathrm{m}$. rectus femoris for skilled players in the context of special exercises". Pedagogics, Psychology, Medical-biological Problems of Physical Training and Sports, vol. 17, pp. 92-98, 2013. DOI: 10.6084/m9.figshare.747479.

[8] Buchanan T.S., Lloyd D.G., Manal K., Besier T.F., "Neuromusculoskeletal modeling: estimation of muscle forces and joint moments and movements from measurements of neural command". Journal of Applied Biomechanics, vol. 20, no. 4, pp. 367-395, 2004. URL: https://www.ncbi.nlm.nih.gov/pmc/articles/PMC1357215/.

[9] Girard O., Nybo L., Mohr M., Racinais S., "Plantar flexor neuromuscular adjustments following match-play football in hot and cool conditions". Scandinavian Journal of Medicine and Science in Sports, vol. 25, pp. 154-163, 2015. DOI: $10.1111 / \mathrm{sms} .12371$

[10] Sandoval A.E., "Electrodiagnostics for low back pain". Physical Medicine and Rehabilitation Clinics of North America, vol. 21, pp. 767-776, 2010. DOI: 10.1016/j.pmr.2010.06.007.

[11] Behm D.G., Whittle J., Button D., Power K., "Intermuscle differences in activation". Muscle and Nerve, vol. 25, pp. 236-243, 2002. DOI: 10.1002/mus. 10008.

[12] Cifrek M., Medved V., Tonkovic S., Ostojic S., "Surface EMG based muscle fatigue evaluation in biomechanics". Clinical Biomechanics, vol. 24, pp. 327-340, 2009. DOI: 10.1016/j.clinbiomech.2009.01.010.

[13] Hibbs A.E., Thompson K.G., French D.N., Hodgson D., Spears I.R., "Peak and average rectified EMG measures: Which method of data reduction should be used for assessing core training exercises?" Journal of Electromyography and Kinesiology, vol. 2, pp. 102-111, 2011. DOI: 10.1016/j.jelekin.2010.06.001.

[14] Hopkins W.G., "Measures of reliability in sports medicine and science". Sports Medicine, vol. 30, pp. 375-381, 2000. DOI: 0112-1642/00/0007-0001. 
[15] "Computer electricneuromyograph M-Test", http://www.dx-systems.biz-gid.com/products/unit?pid=1958 26 (accessed Oct. 18, 2020).

[16] "Skintact diagnostic resting ecg electrodes", http://www.skintact.com/50.0.html (accessed Oct. 18, 2020).

[17] Shrout P.E., Fleiss J.L., "Intraclass correlation: uses in assessing rater reliability. Psychological Bulletin, vol. 86, pp. 420-428, 1979. DOI: 10.1037/0033-2909.86.2.420.

[18] Zanevskyy I., Zanevska L., "Intraclass correlation test-score reliability of a single trial". Journal of Testing and Evaluation, vol. 45, pp. 1073-1080, 2017. DOI: 10.1520/JTE20150485.

[19] Balshaw T.G., Fry A., Maden-Wilkinson T.M., Kong P.W., Folland J.P., "Reliability of quadriceps surface electromyography measurements is improved by two vs. single site recordings". European Journal of Applied Physiology, vol. 117, pp. 1085-1094, 2017. DOI: 10.1007/s00421-017-3595-z.

[20] Fife D.A., Mendoza J.L., Terry R., "The assessment of reliability under range restriction: a comparison of alpha, omega, and test-retest reliability for dichotomous data". Educational and Psychological Measurement, vol. 72, no. 5, pp. 862-888, 2012. DOI: 10.1177/0013164411430225.

[21] Akınoglu B., Kocahan T., "Comparison of muscular strength and balance in athletes with visual impairment and hearing impairment". Journal of Exercise Rehabilitation, vol. 14, pp. 765-770, 2018. DOI: 10.12965/jer.1836304.152.

[22] Lariviere C., Gagnon D., Gravel D., Arsenault A. B., "The assessment of back muscle capacity using intermittent static contractions. Part I: Validity and reliability of electromyographic indices of fatigue". Journal of Electromyography and Kinesiology, vol. 18, no. 6, pp. 1006-1019, 2008. DOI: 10.1016/j.jelekin.2007.03.012.

[23] Mohamed M. N., Azhar A. H. "Postural sway and shooting accuracy of skilled recurve archers". Movement, Health and Exercise, vol. 1, no. 1, pp. 49-60, 2012. DOI: 10.15282/MOHE.V1I0.5.

[24] Huang H. J., Ferris D. P., "Upper and lower limb muscle activation is bidirectionally and ipsilaterally coupled". Medicine and Science in Sports and Exercise, vol. 41, no. 9, pp. 1778-1789, 2009. DOI: 10.1249/MSS.0b013e31819f75a 7.

[25] Franz J. R., Kram R., „How does age affect leg muscle activity/coactivity during uphill and downhill walking?" Gait and Posture, vol. 37, no. 3, pp. 378-384, 2012. DOI: 10.1016/j.gaitpost.2012.08.004.

[26] Lu C., Al-Amri M., „Effects of self-paced incline treadmill walking on lower limb muscles activation level". The XXVII Congress of the International Society of Biomechanics, Calgary, Canada, 31 July -4 August 2019, p. 1. URL: https://www.researchgate.net/publication/335062757_Effect s of self-paced incline treadmill walking on lower limb _muscles_activation_level (accessed Oct. 18, 2020).

[27] Ertan H., "Muscular activation patterns of the bow arm in recurve archery". Journal of Science and Medicine in Sport, vol. 12, no. 3, pp. 357-360, 2009. DOI: 10.1016/j.jsams.2008.01.003.

[28]Ertan H., Soylu A. R., Korkusuz F., "Quantification the relationship between FITA scores and EMG skill indexes in archery". Journal of Electromyography and Kinesiology, vol. 15 , no. 2, pp. 222-227, 2005. DOI: 10.1016/j.jelekin.2004.08.004

[29] Goodwin P.C., Koorts K., Mack R., Mai S., Morrissey M.C., Hooper D.M., "Reliability of leg muscle electromyography in vertical jumping". European Journal of Applied Physiology and Occupational Physiology, vol. 79, pp. 374-378, 1999. DOI: 10.1007/s004210050523.

[30] Koh D., Zainudin N.H., Zawi M.K, "Validity and reliability of the modified physical activity questionnaire for adolescents (PAQ-A) among Malaysian youth," International Journal of Human Movement and Sports Sciences, vol. 8, no. 6, pp. 355 - 360, 2020. DOI: 10.13189/saj.2020.080606.

[31] Gardner J.K, Chia J.T., Miller K.L., "Leg-drive does not affect upper extremity muscle activation during a bench press exercise". International Journal of Human Movement and Sports Sciences, vol. 7, no. 1, pp. 12 - 17, 2019. DOI: 10.13189/saj.2019.070103. 